\title{
Development of Algorithm for Identification of area for Maximum Coverage and Interference
}

\author{
Authors \\ Janak Gupta ${ }^{1}$, Pankaj Kumar ${ }^{2}$ \\ Email-gjuhi.01@gmail.com,unpankaj@gmail.com
}

\begin{abstract}
:
In this paper we consider the following problem: Given a set $n$ shops of Storel in the plane P than how many minimum shops of Store2 to be open for the maximum coverage and interference Store1 Shops. The problem is solve using the Stabbing disk induced by points on the plane P. For the point set P in general position, an upper bound of the stabbing set of $\left(\begin{array}{l}n \\ 2\end{array}\right)$ disks induced by each pair of points a,b $P$ as the diameter of the disk is 2n-2-k. For a point set $P$ in convex position, derive an upper bound on the size of the stabbing set of $\left(\begin{array}{l}n \\ 2\end{array}\right)$ disks induced by each pair of points $a, b \in P$ as the diameter of the disks is $n-2$.

Keywords: Stabbing, Proximity Graph, Gabriel Graphs, Triangulation, Neighborliness, Convex hull.
\end{abstract}

\section{INTRODUCTION}

Now the there is a trend of brands store either we are talking about clothing, sweets or any items. So, it is very difficult for owner of the new brand chains where to open the store in the same market where already the well established brand store are exists.

Consider a city having the multiple shops of shopping chain, whose shops are called the Store. It is common that the customer will prefer to go the nearest store to them (assuming that all brand store having the same quality product)

Now consider that a new brand owner wants to setup the chain of shops called Store2 at the same area where the Store 1 are already exists. What are the best locations for Store2 so, Store2 can "interfere" the business of Store1 as much as possible, sometimes using same number of shops or sometimes using minimum number of shops possible. There may be different criteria on how to interfere their business. Note that, knowingthat some opponent may place their shops in future, Store1 also had placed his shops in the optimal ways, and the problem is to fond the optimal places for the Store2. The paper is all about the optimal solution of above problem using the stabbing disk induced by points on the plane. Let the number of Store1 are ' $n$ ' with point set P.Initally to get the solution of the problem the disk is defined by the pairs of points as diameter.

For the neighbourliness concept proximity are used Proximity graph can be intuitively defined as follows: given a point $\mathrm{P}$ in the plane, the vertices of the graph $\mathrm{s}$, there is an edge between a pair of vertices $\mathrm{p}, \mathrm{q} \in \mathrm{P}$ if they satisfy some particular notion of neighbourliness.

Proximity graphs can be used in shape analysis and in data mining ${ }^{[7][10]}$. In graph drawing, a problem related to proximity graphs is to find the classes of graphs that admit proximity drawing for some notion of proximity, and whenever possible to efficiently decide, for a given graph, whether such a drawing exists ${ }^{[4][8]}$.

For the notation of neighbourliness of the pair of vertices $a, b$ is the closed disk $D_{a b}$ with diameter $\overline{a b}$ is the Gabrial graphs. An edge $a b$ is in the Gabriel graph of a point set $\mathrm{P}$ if and only if $P \cap D_{a b}=\{a, b\}$ (see fig.1) ${ }^{[1]}$. Gabriel graphs were introduced by Gabriel et al. ${ }^{[6]}$ in the context of geographic variation analysis.

In the case of Delaunay Graphs.DG(P) ${ }^{[2]}$, the region of influence of a pair of vertices $a, b$ is the set of closed disks $\mathrm{D}_{\mathrm{ab}}$ with chord $\overline{a b}$. An edge $\mathrm{ab}$ is in the Delaunay Graph of a point set $\mathrm{P}$ if and 
only if there exists a disk dabe $D_{a b}$ such that $\mathrm{P} \cap \mathrm{D}_{\mathrm{ab}}=\{\mathrm{a}, \mathrm{b}\}$.
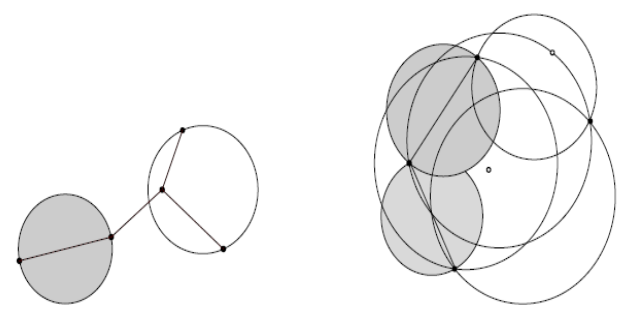

Fig.1: Gabriel graph.

The vertices defining a shaded disk are adjacent and the pairs defining the shaded disks are adjacent because their disk does not contain any other vertex, in contrast to the other vertices defining the shaded disk .Right: Witness Gabriel graph .Black points are the vertices of the graph, white points are the witnesses.Each pair of vertices defining a shaded disk are adjacent and the pairs defining the unshaded disks are not.

\section{STABBING DISKS INDUCED BY POINTS ON THE PLANE}

Let $\mathrm{P}$ be a set of $\mathrm{n}$ points, and let $\mathrm{S}$ be the set of $\left(\begin{array}{l}n \\ 2\end{array}\right)$ disks induced by each pair of points $a, b \in P$. The diameter of the disk is defined by the line segment $\overline{a b}$.

Sufficiency of Points for Stabbing In this section, we will prove that for $\mathrm{n}$ points in general position, the size of the stabbing set is bounded above by $2 n-2-k$, where $k$ is the number of points on the boundary of the convex hull of the points of $\mathrm{P}$.

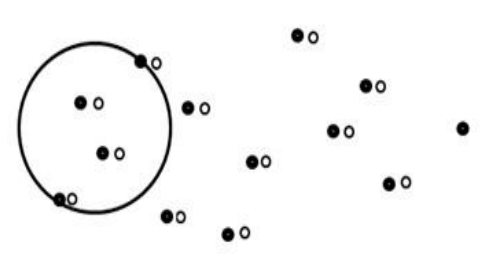

Fig. 2: Points in $\mathrm{P}$ are show with filled dots and the stabbing points are hown using dots whose interiors are empty.

The earlier bound on stabbing set was due to Aronov et al ${ }^{[1]}$ which we state here.

Theorem 2.1. ${ }^{[1]} n-1$ witnesses are always sufficient to stab all the disks in S.
Proof. We argue the upper bound here. Without loss of generality, assume no two points of $\mathrm{P}$ lie on the same vertical lines, this can be achieved by an appropriate rotation of the cooradinate system. Put a witness slightly to the right of each point of $\mathrm{P}$, except for the right most one (see Fig. 2). Every disks with diameter determined by two points of $\mathrm{P}$ will contain a witness. We now state a few lemmata that would be useful in proving our bound.

Lemma 2.2. A point sees the diameter of a circle with an angle greater than $\pi / 2$ if and only if the point lies inside the circle.
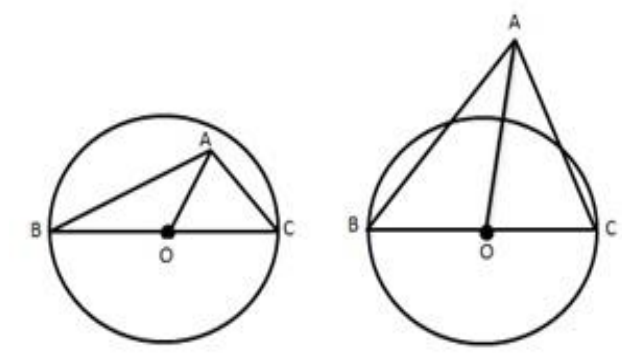

Fig. 3: Left: Point sees diameter with an angle greater than $\pi / 2$. Right:Point sees diameter with an angle less than $\pi / 2$.

Proof. $[\Rightarrow$ ]Let the point A lies inside the circle. We will prove that $\angle B A C>\pi / 2$. See fig. 3 (left).

Let $\angle \mathrm{OAC}=\alpha, \angle \mathrm{OAB}=\beta, \angle \mathrm{ACO}=\gamma \angle \mathrm{ABO}=\delta$.

As the point $\mathrm{A}$ lies inside the circle, $\mathrm{OC}>\mathrm{OA}$ and $\mathrm{OB}>\mathrm{OA}, \Rightarrow \alpha>\gamma$ and $\beta>\delta$.

In $\triangle \mathrm{AOB}, \angle \mathrm{AOB}+\angle \mathrm{ABO}+\angle \mathrm{OAB}=\pi$

$\Rightarrow \alpha+\beta+\gamma+\delta=\pi$

$\Rightarrow 2(\alpha+\beta)>\pi, \alpha+\beta>\pi / 2$.

$\Rightarrow \angle \mathrm{BAC}=\alpha+\beta>\pi / 2$.

$[\Leftarrow]$ Now, to prove the only if part we will prove that if a point lies outside the circle than it eill see the diameter of the circle with an angle $<\pi / 2$. See fig. 3(right).

As the point A lies outside the circle, $\mathrm{OC}<\mathrm{OA}$ and $\mathrm{OB}<\mathrm{OA}, \Rightarrow \alpha<\gamma$ and $\beta<\gamma$

$\Rightarrow \alpha+\beta<\gamma+\delta, \angle \mathrm{AOB}=\alpha+\gamma$.

In $\triangle \mathrm{AOB} . \angle \mathrm{AOB}+\angle \mathrm{ABO}+\angle \mathrm{OAB}=\pi$

$\Rightarrow \alpha+\beta+\gamma+\delta=\pi$

$\Rightarrow 2(\alpha+\beta)<\pi \quad \alpha+\beta<\pi / 2$

$\Rightarrow \angle \mathrm{BAC}=\alpha+\beta<\pi / 2$ 
So we have proved that a point will see the diameter of a circle with an angle greater than $\pi / 2$ if and only if the point lies inside the circle.

So by lemma 2.2, a point will stab a disk if and only if it sees the diameter of the disk with an angle greater than $\pi / 2$.

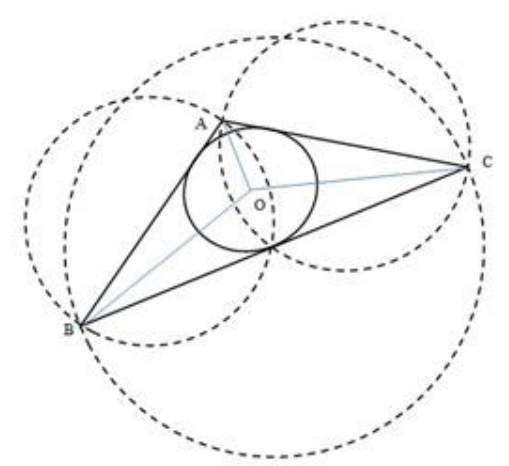

Fig.4:Incenter sees the sides of the triangle with an angle greater than $\pi / 2$.

Lemma 2.3. The intersection of three disks having the sides of a triangle as their diameter is always non empty.

Proof. To prove that the intersection of three disks is non-empty, we need to prove that there is at least one point which sees all the three sides of the triangle with greater than $\pi / 2$.

Let $\mathrm{O}$ be the in center of the triangle $\mathrm{ABC}$ (see Fig. 4).

$\angle \mathrm{OAC}+\angle \mathrm{OCA}<\pi / 2($ as $\angle \mathrm{BAC}+\angle \mathrm{BCA}<\pi)$

$\Rightarrow \angle \mathrm{AOC}>\pi / 2$

This implies that $\mathrm{O}$ sees the side $\mathrm{AC}$ with an angle greater than $\pi / 2$ or in other words $O$ lies inside the disks having AC as its diameter. Similarly, we can prove that $\mathrm{O}$ lies inside the disks corresponding to side $\mathrm{AB}$ and $\mathrm{BC}$.

Lemma 2.4. For any $\triangle \mathrm{ABC}$, let $\mathrm{E}$ be a point on any side of $\triangle \mathrm{ABC}$ (say $\mathrm{BC}$ ) (see Fig. 5). The disks corresponding to diameter $\mathrm{AE}$ (vertex $\mathrm{A}$ is opposite to side BC) contains the common intersection region of disks having the side of $\triangle \mathrm{ABC}$ as their diameters.

Proof. For any point $\mathrm{O}$ that lies in the common intersection of three disks corresponding to the sides of the $\triangle \mathrm{ABC}$ (see Fig. 5), there will be the following three cases.

The point $\mathrm{O}$ will lie on the line segment AE.
The point $\mathrm{O}$ will lie on the line segment $\mathrm{AE}$. The point $\mathrm{O}$ will lie to the right of the line segment AE.

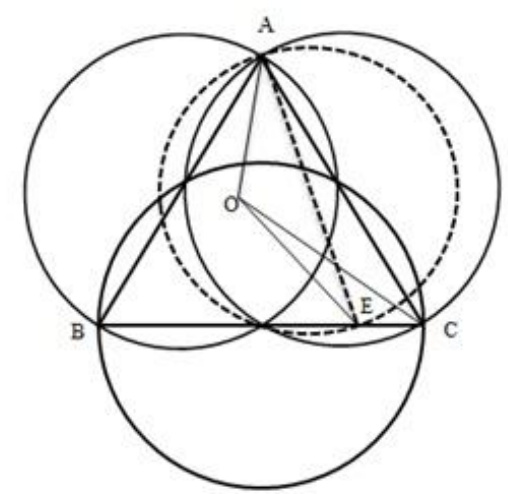

Fig.5: Disks corresponding to $\mathrm{AE}$ contains the common region of disks corresponding to $\mathrm{AB}, \mathrm{BC}$ and CA.

In the first case, when the point $\mathrm{O}$ lies on $\mathrm{AE}$, it is trivial to show it will lie in the disks with $\mathrm{AE}$ as diameter. In the second case, when the point $\mathrm{O}$ lies to the left of $\mathrm{AE}, \angle \mathrm{AOE}$ will be equal to the sum of $\angle \mathrm{AOC}$ and $\angle \mathrm{EOC}$ (see Fig. 5), which will be greater than $\pi / 2$, as $\angle A O C>0$. This implies that point $\mathrm{O}$ lies inside the disk corresponding to AE. The third case is similar to the second case.

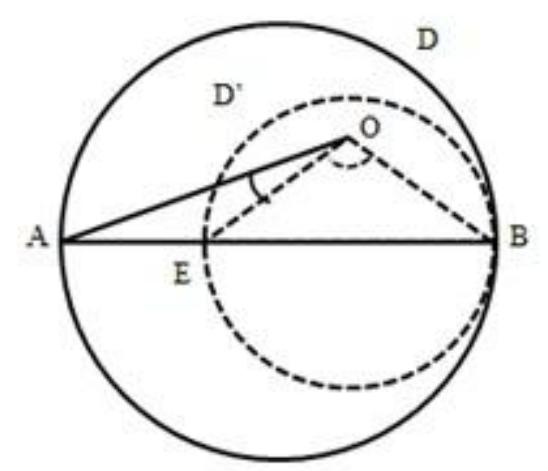

Fig.6: Showing that D' lies completely inside D.

Lemma 2.5. Let $D$ be a disk with $A B$ as its diameter (see Fig. 6). The disk D' with diameter $\mathrm{EB}$, where $\mathrm{E}$ is a point that lies on $\mathrm{AB}$, lies completely inside D.

Proof. To prove that D' will lie completely inside $\mathrm{D}$, we will show that any point $\mathrm{O}$ that lie inside $\mathrm{D}^{\prime}$ sees $\mathrm{AB}$ with an angle greater than $\pi / 2 . \angle \mathrm{AOB}=$ $\angle \mathrm{AOE}+\angle \mathrm{EOB}$ (see Fig. 6). $\angle \mathrm{AOB}$ will be greater than $\pi / 2$, as $\angle \mathrm{EOB}>\pi / 2$ and $\angle \mathrm{AOE}>0$. 


\section{Triangulation of a Planar Point Set}

Let $\mathrm{P}$ be a set of $\mathrm{n}$ points in the plane. To be able to formally define a triangulation of $\mathrm{P}$, we first define a maximal planar subdivision as a subdivision $\mathrm{S}$ such that no edge connecting two vertices can be added to $S$ without destroying its planarity. In other words, any edge that is not in $S$ intersects one of the existing edges.

Definition 1. A triangulation of $P$ is defined as a maximal planar subdivision whose vertex set is $\mathrm{P}$.

Lemma 2.6.[3], Let $P$ be a set of $n$ points in the plane, not all collinear, and let $\mathrm{k}$ denote the number of points in $\mathrm{P}$ that lie on the boundary of convex hull of $\mathrm{P}$. Then any triangulation of $\mathrm{P}$ has $2 n-2-k$ triangles and $3 n-3-k$ edges.

Proof. Let $\tau$ be triangulation of $\mathrm{P}$, and let $\mathrm{m}$ denote the number of triangles of $\tau$ note that the number of faces of triangulation, which we denote by $n_{f}$. is $\mathrm{m}+1$. Every triangle has three edges, and the unbounded faces has $\mathrm{k}$ edges. Furthermore. Every edges is incident to exactly two faces. Hence, the total number of edges of $\tau$ is $n_{e}=(3 \mathrm{~m}+\mathrm{k}) / 2$. Euler's formula tells us that $\mathrm{n}-\mathrm{n}_{\mathrm{c}^{-}}$ $\mathrm{n}_{\mathrm{f}}=2$ plugging the values of $\mathrm{n}_{\mathrm{c}}$ and $\mathrm{n}_{\mathrm{f}}$ into the formula, we get $m=2 n-2-k$ which in turn impies $n_{e}=3 n-3-k$.

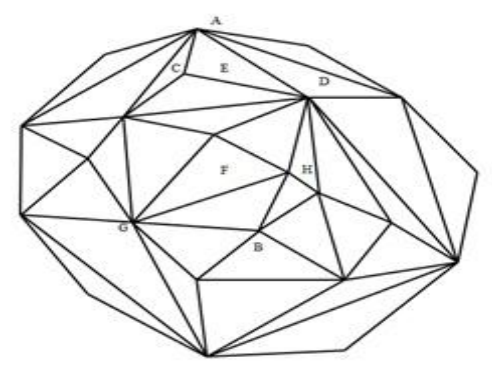

Fig.7: 2n-2-k points are sufficient to stab all the disks

Theorem2.7. let $\mathrm{P}$ be asset of $\mathrm{n}$ points in the plane in general position, and let $\mathrm{k}$ denote the number of points in $p$ that lie on the boundary of the convex hull of $\mathrm{P}$. then the size of for stabbing set for $\mathrm{P}$ is bounded above by $2 \mathrm{n}-2-\mathrm{k}$.

Proof: firest we find a triangulation $\mathrm{T}$ of the point set P. According to lemma 2.6, there will be 2n-2$\mathrm{k}$ triangles in $\tau$. then for each triangle we put a stabbing point at the in center of the triangle. So by lemma 2.3 , these stabbing points will stab all the disks having any edge of the triangulation as its diameter. Now, for the points A and B (see Fig. 7), which are not adjacent in the triangulation of $\mathrm{P}$, if we draw an edge $A B$, that edge will intersect one of the opposite side $\mathrm{CD}$ of $\mathrm{A}$ at point $\mathrm{E}$ and the opposite side $\mathrm{GH}$ of $\mathrm{B}$ at $\mathrm{F}$ in $\tau$ (see fig.7). now according to lemma 2.4 the disk with diameter $\mathrm{AE}$ will be stabbed by the stabbing point of triangle ACD and by lemma 2.5, the disk with diameter $\mathrm{AB}$ is stabbed as the point $\mathrm{E}$ lies on AB.so we have stabbed all the disk with $2 n-n-k$ stabbing points, this completes the proof.

Corollary2.8. let $\mathrm{P}$ be a set of $\mathrm{n}$ points in the plane in convex position. Then all size of stabbing set for $\mathrm{P}$ is bounded above by $\mathrm{n}-2$.

Proof. If the points of $\mathrm{P}$ are in convex position, then we can triangulate $\mathrm{P}$ with $\mathrm{n}-2$ triangles (see fig. 8), and then follow the proof of theorem 2.7 to prove the sufficiency of $n-2$ stabbing points.

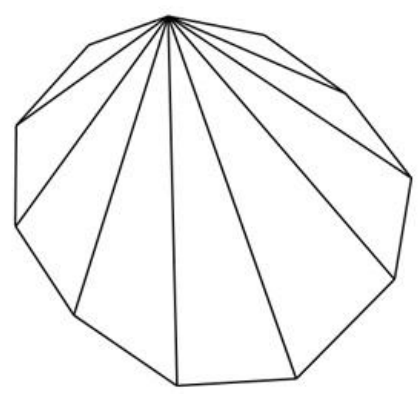

Fig.8: For the points in convex position there will be $n-2$ triangles in any triangulation

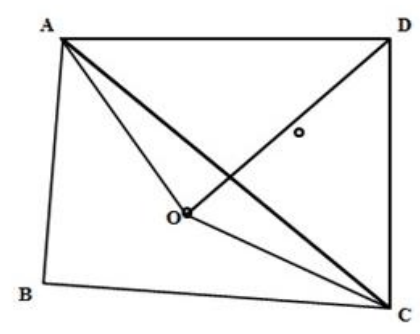

Fig.9: Two points are necessary to stab all the disks corresponding to the edges of a quadrilateral 2.2.2 Necessity of points for stabbing

Theorem2.9. Two stabbing points are necessary to stab all the disks for a point se $\mathrm{P}$ having four points. 
Proof. Let the four points A, B, C and D from a quadrilateral, and then there are two stabbing points corresponding to $\triangle \mathrm{ABC}$ and $\triangle \mathrm{ACD}$ (see fig. 9).To prove the necessity of two stabbing points, it is sufficient to prove that any one of these two points cannot stab all the disks corresponding to the sides of the quadrilateral, Let $\mathrm{O}$ be the stabbing point which stabs the disks with diameters $\mathrm{AB}, \mathrm{BC}$ and $\mathrm{AC}$ (fig.9), we prove that $\mathrm{O}$ cannot stab both the disks with diameters $\mathrm{AD}$ and $\mathrm{CD}$ as follows: $\angle \mathrm{AOC}$ will be strictly less than $\pi$, this implies that either $\angle A O D$ or $\angle C O D$ will be strictly less than $\pi / 2$. So O cannot stab both the disks. Similarity, we can prove that the stabbing point lies inside the $\triangle \mathrm{ACD}$ can not stab the the disks with diameter as $\mathrm{AB}$ and $\mathrm{BC}$.

\section{RESULT}

For the $\mathrm{n}$ points in the plane $\mathrm{P}$ and itprofess in the lemma 2.6 that for the $\mathrm{k}$ points lies on the boundary of convex hull in the plane Phas the $2 n-$ $2-\mathrm{k}$ triangles and the $3 \mathrm{n}-3-\mathrm{k}$ edges. Each triangle has the single stabbing point. If all the $\mathrm{n}$ points are at convex position in the plane than the number of stabbing points are the $\mathrm{n}-2$ for set $\mathrm{P}$.

\section{CONCLUSION}

As it is discuss in the paper that as the problem of finding the stabbing sets for disks it is claim the upper bound of stabbing sets for disks induced by points on the plane as diameter, as 2n-2-k.Tthis bound is batter (minimum) than the previous bound given by Aronov et al. When the points are in the convex position the number of stabbing points are $n-2$.

Now it is clear that when $n$ shops of Store 1 are in the plane $\mathrm{P}$ having $\mathrm{k}$ shops at the convex hull position than the minimum number of shopsis $2 n-$ 2-k of Store 2 are the best to be open and when all the shops are at convex position than the best number of shops is $n-2$. So,Store 2 will covers the maximum area and easily interrupt the Store 1.

\section{REFERENCES}

1. Boris Aronow, Muriel Dulieu, and ferran Hurtado, "Witness Gabriel graphs", 2010.

2. Boris aronov, Muriel dulieu, and ferranhurtado, "Witness Delaunay graphs", Computational geometry: theory and application 44:329:344,2011.

3. M.d.berg, o.cheong, M. kreveld, and M.overmars, "Computational geometry", Algorithms and application springer 3rd edition, 2008.

4. G. Di Battista, p. edes, R. tamassia, and I.G. Tollis., "Graph drawing. Algorithms for the visualization of graphs", Prentice hall, 1999.

5. Robert J. Fowler, Mike Paterson and steven L. Tanimoto, "Optimal packing and covering in the plane are $n p$ complete",Inf process. Lett.,12(3):133137, 1981.

6. K. r. Gabriel and R.r.sokal, "A new statistical approach to geographic variation analysis", Systematic Zoology, 18:259278, 1969.

7. J. w. jaromczyk and G.t. Toussaint, "Relative neighbourhood graphs and their relatives", Proc. IEEE, 80:1502-1517, 1992.

8. G. Liotta, "Proximity drawings", In inrr. Tamassia, editor, handbook of graphs drawing ndvisualization . CRC press.

9. Frank Nielsen, "Fast stabbing of boxes in high dimension", Theoretical computer science, 246(1/2):53-75, 2000.

10. G. T. Toussaint,"Geometric proximity graphs for improving nearest neighbour methods in instance- based learing and data mining",International Journal of Computer Geom. And Applications. 15(2).

11. G.T. Toussaint,"Some unsolved problems on proximity graphs", 1991 